\title{
Belgeo
}

Revue belge de géographie

$1 \mid 2002$

Miscellaneous : Africa

\section{Croyances coutumières, pratiques foncières et développement rural au Togo. Cas des préfectures de Haho et du Moyen-Mono}

Traditional beliefs, land distribution practices and rural development in Togo.

The case of the Haho and Moyen-Mono prefectures

\section{Selom K. Klassou}

\section{OpenEdition \\ Journals}

Édition électronique

URL : https://journals.openedition.org/belgeo/15437

DOI : 10.4000/belgeo.15437

ISSN : 2294-9135

\section{Éditeur :}

National Committee of Geography of Belgium, Société Royale Belge de Géographie

\section{Édition imprimée}

Date de publication : 30 mars 2002

Pagination : $29-44$

ISSN : 1377-2368

Référence électronique

Selom K. Klassou, « Croyances coutumières, pratiques foncières et développement rural au Togo. Cas des préfectures de Haho et du Moyen-Mono », Belgeo [En ligne], 1 | 2002, mis en ligne le 31 mars 2002, consulté le 14 juin 2021. URL : http://journals.openedition.org/belgeo/15437 ; DOI : https://doi.org/ $10.4000 /$ belgeo. 15437

Ce document a été généré automatiquement le 14 juin 2021.

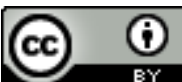

Belgeo est mis à disposition selon les termes de la licence Creative Commons Attribution 4.0 International. 


\section{Croyances coutumières, pratiques foncières et développement rural au Togo. Cas des préfectures de Haho et du Moyen-Mono}

Traditional beliefs, land distribution practices and rural development in Togo. The case of the Haho and Moyen-Mono prefectures

Selom K. Klassou

1 La terre joue dans la vie des populations rurales un rôle inestimable. Elle intervient dans les activités quotidiennes des familles et, en tant que support des cultures, donc par sa fonction nourricière, elle est considérée par les populations adja et éwé qui habitent respectivement les préfectures du Moyen-Mono et de Haho au Togo (fig. 1) comme source et garante de la vie ou, mieux encore, comme la « vie même ». La terre a aussi un caractère sacré très prononcé dans les secteurs de peuplement ancien comme les cantons de Tado et de Tohoun et la région de Notsé, par exemple, où elle apparaît même comme une divinité à laquelle on offre des sacrifices. 
Figure 1. Les préfectures de Haho et du Moyen-Mono au Togo.

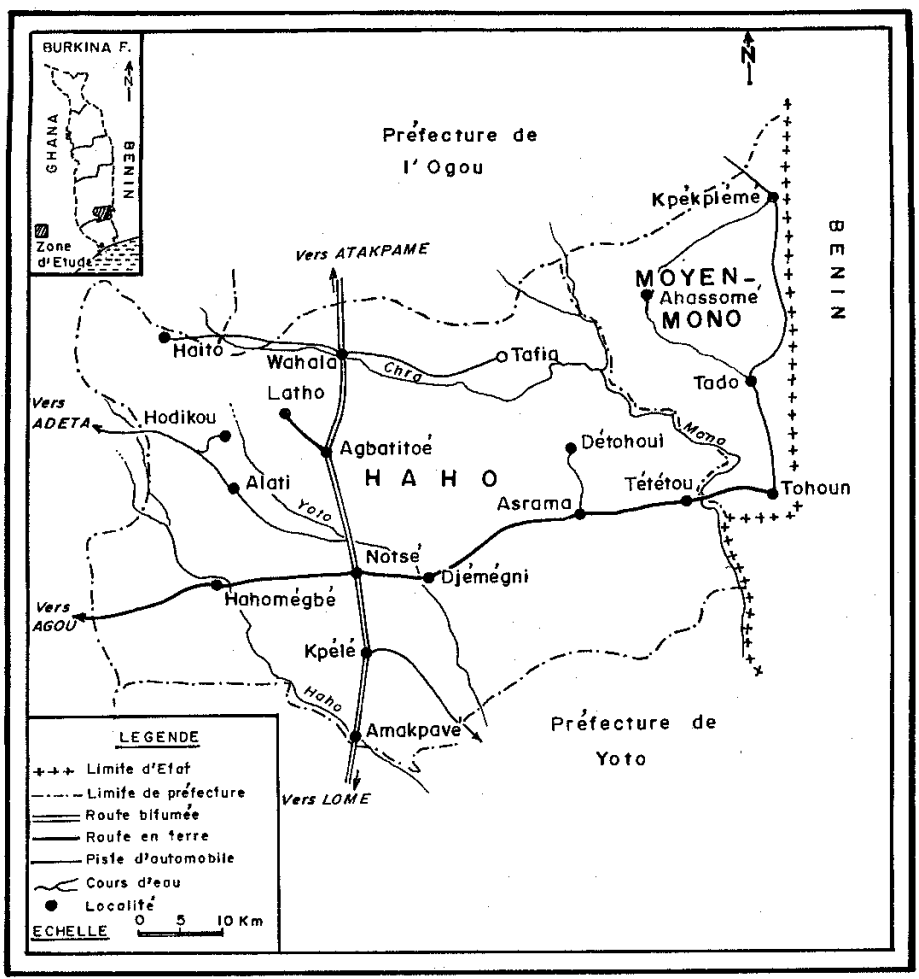

2 Pendant longtemps marquée par ces considérations, la terre était distribuée gratuitement et exploitée collectivement dans le cadre de l'économie familiale traditionnelle. Mais depuis l'introduction du système colonial et surtout depuis l'indépendance, elle est l'objet d'une évolution remarquable tant pour le concept de propriété que pour les modes de son acquisition. Les conséquences de cette évolution sur le développement régional sont importantes et très diverses.

\section{Occupation de l'espace et représentation de la terre par les populations adja et éwe du moyen-mono et du haho}

3 La plupart des traditions du Sud-Togo soutiennent que, jadis, l'occupation des terres par les hommes ne se faisait pas seulement en fonction des conditions géographiques du milieu. Elle passait d'abord et avant tout par des «négociations» avec les «forces invisibles » de la nature, qui débouchaient sur des pactes : agriculteurs, pêcheurs et chasseurs concluaient avec les divinités chthoniennes supposées habiter les lieux des pactes telluriques qui les mettaient en accord avec les choses de la nature et leur permettaient de prendre possession du sol. Ces cérémonies furent accomplies par les ancêtres premiers défricheurs et premiers occupants qui, de ce fait, sont devenus les seuls et uniques propriétaires des terres. Leurs esprits restent intimement liés à cellesci. Pour les populations adja et éwé, la terre incarne l'ancestralité.

4 En effet, tout comme dans d'autres régions africaines, la terre avec laquelle les ancêtres ont conclu un pacte ou qui est leur site funéraire constitue et reste leur propriété exclusive. Mais ancestrale, la terre est aussi les ancêtres mêmes, ou même les dieux. 
C'est, bien sûr, ce qui explique que dans tous les villages de la région, des libations sont faites aux mânes des ancêtres lors de tous les actes concernant la terre. Doit-on alors comprendre que les esprits des ancêtres se sont incorporés à la terre? A Tado, on raconte que l'ancêtre fondateur du royaume adja, le premier roi, n'est pas mort de mort naturelle : « la terre s'ouvrit et l'engloutit ». Son nom est d'ailleurs très significatif; il s'appelait, dit-on, « Togbui-Agni », ce qui signifie « l'ancêtre-terre », mais on ne sait s'il portait ce nom dès la naissance ou s'il lui a été attribué après sa mort. Le puissant roi Badja, l'un des successeurs de Togbui-Agni, est mort de la même façon. De l'endroit où il disparut dans la terre a commencé à sourdre une rivière qui porte le nom de Badjamé, c'est-à-dire « rivière de Badja ».

5 Toujours à Tado, considéré comme origine ancestrale des peuples adja et éwé, le souverain qui est intronisé doit rituellement conquérir la terre, source divine de la vie, par des cérémonies "d'ascension" d'une colline surplombant le paysage, afin de maîtriser les forces occultes qu'elle recèle et de les utiliser au mieux pour le bien-être de ses sujets. On lui prête alors des pouvoirs de démiurge qui lui confèrent aux yeux des foules une puissance redoutable. Autour de sa personne est développé le sens du sacré : il est à la fois prêtre de la terre et monarque séculier, véritable monument, personnage vénéré, objet de culte. Il peut éloigner les guerres, les épidémies et les famines, faire pleuvoir en temps de sécheresse et arrêter la pluie en cas d'inondation, faire fructifier le sol et les récoltes, guérir la stérilité des femmes, etc. (Gayibor, 1990). On lui attribue donc des pouvoirs surnaturels immenses, ce qui justifie le grand prestige dont il jouit et surtout sa forte influence sur l'organisation de la société et des activités socioéconomiques en milieu adja.

6 On le voit, la terre est la base culturelle magico-religieuse du pouvoir du roi adja et du roi éwé. Les souverains du pays adja-éwé portent d'ailleurs le titre de « roi de terre »: Anyigbanfio à Tado et Anyigbafia à Notsé.

7 La terre a un caractère sacré qui, on s'en doute, s'accompagne d'interdits stricts parmi lesquels on citera celui de la souillure de la terre par le sang humain (meurtre violent), celui de l'inobservance des accords conclus avec les puissances chthoniennes, celui du non-respect des sites «d'ascension rituelle ». On comprend pourquoi, dans les années 1980, le roi Adjakanumabou (décédé en 1999) avait tenté (mais sans cependant y parvenir) de faire démolir des maisons d'habitation du quartier de Bétuimé à TadoDomé parce qu'elles avaient été construites sur la colline de son « ascension royale ».

8 Les pratiques relatives à la terre décrites ci-dessus ne sont pas uniquement symboliques; elles sont plutôt très culturelles et révèlent les croyances des populations de la région et les rapports qu'elles entretiennent avec leur environnement. A bien des égards, ces pratiques inscrivent l'histoire sur la terre d'autant plus que chaque nouveau roi doit «monter» une colline différente de celle de ses prédécesseurs, colline à laquelle reste lié son nom ; le paysage naturel est donc marqué de signes dont la lecture restitue l'histoire.

9 Comme on le voit, les populations adja et éwé entretiennent avec la terre un type de rapport particulier. Celui-ci fait d'elle un bien très précieux. Au dire de la population, c'est le bien le plus cher que l'on puisse avoir, mis à part l'enfant. Nourricière, source de vie, la terre assure aussi un lien intrinsèque avec les forces invisibles de l'au-delà, elle est comme le cordon ombilical qui permet aux collectivités de communiquer avec leurs ancêtres. Ne pas en avoir signifie perdre son identité, perdre ses repères. La détention du droit sur la terre est source d'intégration dans la communauté 
villageoise ; elle est le signe d'une « autochtonie » qui est très recherchée en raison du fait qu'elle confère de nombreux avantages : droit de pratiquer les cultures arbustives, droit de prendre la parole dans certaines assemblées, droit de se faire élire chef, c'est-àdire droits dont ne peuvent disposer les étrangers.

10 Support des cultures, la terre est aussi source de richesse. La possession de grandes étendues confère un prestige certain. Jadis, l'igname était la principale culture alimentaire de la région et les lignages disposant de terres adaptées à cette culture étaient très sollicités et très courtisés dans les assemblées de village. Il leur suffisait de disposer de bras robustes et nombreux pour s'assurer des productions relativement importantes si les conditions climatiques avaient été favorables, et organiser ainsi en fin d'année de gigantesques ripailles au cours desquelles pouvaient manger et boire plusieurs centaines de convives. Le fait que des membres d'autres lignages et que des étrangers avaient recours à eux pour avoir des parcelles de terre et leur vouaient pour ce fait une considération exceptionnelle leur conférait aussi un grand honneur.

11 Tout cela est à l'origine d'un très fort attachement à la terre qui se manifeste par de multiples offrandes aux divinités telluriques et en particulier par le fait qu'elle est très convoitée et qu'on s'efforce de la préserver par tous les moyens pour sa descendance, parfois au prix d'âpres luttes au cours desquelles on n'hésite pas à recourir aux esprits maléfiques contre ses adversaires, particulièrement par le canal des envoûtements. La majorité des conflits ouverts ou latents qui minent la vie dans les villages découlent de revendications territoriales.

12 Le caractère divin et sacré de la terre, son ancestralité et surtout le fait que le règlement des problèmes fonciers se fait ici, comme on le constate dans d'autres régions du monde, par exemple en Australie, «sur la base de généalogies et de témoignages retraçant les activités des ancêtres transmises par l'histoire orale pour attester l'ancienneté de l'occupation d'une terre par telle ou telle famille" (Glowczewski-Barker, 1992, p. 91), l'usage dans les conflits de terre de forces invisibles dont seules les personnes âgées détiennent le secret, ont conduit au principe de la gestion de la terre par les vieux. Il en est résulté, en droit foncier, la constitution de propriétés lignagères ou de propriétés relevant des grandes familles d'un lignage. Elles sont sous la responsabilité des chefs de lignage ou des chefs de ces grandes familles.

\section{Le régime de la terre et le concept de propriété}

Traditionnellement, la terre étant la propriété des ancêtres, elle est donc pour les générations actuelles une propriété collective, en principe inappropriable par les individus. Il y a « copossession dans l'indivision » comme l'écrit Lombard (1961, p. 197). Le chef de lignage détient le pouvoir de contrôle sur la propriété de sa lignée. La société adja-éwé est socialement structurée de telle façon que toutes les familles se réclamant d'un ancêtre unique forment un lignage sous la responsabilité du plus vieux personnage de celui-ci qui, en raison de la sagesse qu'on lui prête généralement, en est le chef : il administre les mariages, dirige les cérémonies de naissance et de décès ainsi que les libations aux dieux et aux ancêtres. Dans le domaine foncier, c'est encore naturellement lui qui, aidé par les autres anciens du lignage, joue un rôle fondamental dans la connaissance des droits, veille à l'intégrité des terres de son lignage et assure la répartition des usages. Il peut aussi accorder un droit de culture à des gens du village extérieurs à son lignage ou même à des étrangers à la communauté villageoise. En effet, 
comme «la terre n'est pas un bien accumulable mais un moyen de survie et de reproduction du groupe social et de ses éléments constitutifs » (Gu-Konu, 1986, p. 246), tous les membres de la communauté, y compris les allochtones, peuvent y avoir accès. Il y a bien sûr quelques différences entre les villages ou même entre les différents lignages au sein du même village, par exemple en ce qui concerne les démarches à accomplir, mais il s'agit au total d'un usufruit respectueux des normes coutumières.

Dans le cadre de l'économie de subsistance ou à faible capacité d'échange dans laquelle évoluaient les sociétés africaines précoloniales, l'exploitation de terres collectives se faisait sans conflit majeur apparent (si on s'en tient aux déclarations recueillies dans nos enquêtes), d'autant plus que les instructions des chefs de lignage étaient exécutées avec un respect religieux et que le travail agricole même se faisait dans un cadre collectif, les jeunes gens, même mariés et disposant d'enfants, étant tenus de travailler dans les champs de leurs parents, c'est-à-dire du père s'il était encore vivant, sinon de l'oncle ou du frère aîné. Le fait d'accomplir les travaux agricoles en groupe était dû aussi, semble-t-il, à l'insécurité. Non seulement on redoutait les fauves (hyène, lion, léopard, buffle, etc.), mais il y avait aussi les guerres, en particulier avec les royaumes d'Oyo (Nigeria) et du Dahomey, et les incursions des États côtiers esclavagistes qui entretenaient la terreur. Dans ce contexte, il était indiqué de travailler en groupe sous la protection des personnes âgées, plus précisément des chefs de famille. Quant à l'absence de conflit majeur à cette époque, elle était liée à l'abondance des terres cultivables. Les domaines fonciers des lignages étaient vastes et fort multiples au point que certains lignages craignaient que des étrangers ne viennent s'emparer de certaines de leurs terres en friche. Ainsi des lignages recherchaient-ils parfois des étrangers ou des gens nécessiteux pour les y installer en leur nom, et en marquer ainsi l'occupation effective

Dans le cadre de l'exploitation des terres collectives, c'était le chef de lignage, encore appelé «chef de terre", qui déléguait le droit d'usufruit aux exploitants. Si ce droit était permanent pour les membres de son lignage, il était (théoriquement) révocable pour les non-membres, qui pouvaient à tout moment se faire retirer leurs parcelles s'ils s'écartaient des prescriptions coutumières. Mais il semble que peu de cas de retrait de parcelle se soient produits, du moins jusqu'au début de la période coloniale. Au contraire, raconte-t-on dans les villages, beaucoup d'exploitants étaient devenus de fait, l'exploitation se prolongeant par les enfants et petits-enfants, «propriétaires » de leurs parcelles.

Le principe des terres lignagères n'a pas résisté aux transformations qui affectent cette région depuis l'époque coloniale. Avec l'accroissement de la population et le développement de l'économie monétaire, les besoins en terre sont devenus plus importants en quantité et en qualité. Les comportements des chefs de lignage ont été de plus en plus critiqués. En particulier, il leur a été reproché de distribuer les droits de culture sur des bases népotistes en attribuant les meilleures terres à leurs proches, et de profiter exagérément du fruit du travail commun d'autant plus que ces patriarches fatigués par l'âge et les travaux accomplis dans leur jeunesse, ne pouvant plus vraiment travailler dans les champs, se contentent d'une sorte de contrôle des activités. Les membres des nouvelles générations pensent que les raisons qui étaient à la base de l'exploitation collective des terres n'existent plus. Ils trouvent plus intéressant pour eux d'entretenir, avec tout le soin possible, des exploitations individuelles dont ils espèrent tirer de quoi acheter les produits manufacturés européens d'importation qui 
les fascinent. C'est dans ce cadre de la recherche d'une autonomie économique et financière qu'à partir des années 1920 et 1930, période caractérisée aussi par l'accélération de l'immigration des Éhoué du Bénin et le début de l'essor des cultures commerciales (coton et arachide) dans les actuelles préfectures du Moyen-Mono et du Haho, les grandes familles traditionnelles ont éclaté en de multiples ménages au sens occidental du terme, et que les exploitations collectives ont été atomisées en exploitations individuelles de petite taille, chaque membre d'un ménage (homme, femme(s) et parfois enfants d'un certain âge) ayant son propre champ qu'il gère librement avec une certaine autonomie économique. Sur le plan foncier, cela s'est traduit d'abord par l'ouverture de champs individuels sur les propriétés collectives puis par l'éclatement des structures traditionnelles et l'appropriation individuelle du sol. On a assisté à la naissance d'une multitude de petites propriétés paysannes sur les ruines des anciens domaines communautaires. Aujourd'hui, seules quelques rares terres de quelques terroirs (Tado, Djadéhoé) peuvent être regardées comme relevant encore de la propriété collective au sens du droit coutumier, comme le montrait en 1989 une étude, à laquelle nous avons participé, de faisabilité d'un projet de construction d'un barrage hydroélectrique sur le fleuve Mono à Adjarala et comme l'ont confirmé les nombreuses enquêtes que nous menons dans la région depuis 1994. Les quelques terres collectives qui existaient encore étaient des domaines en attente d'être partagés.

17 Certes l'individualisme foncier, qui s'est substitué aux structures collectives traditionnelles, n'empêche pas les gens de continuer à s'intégrer dans un ensemble plus vaste, le lignage par exemple pour des cérémonies familiales ou dans des circonstances qui réclament la solidarité des personnes descendant d'un même ancêtre. Dans l'organisation du travail agricole, les pratiques sociales traditionnelles n'ont d'ailleurs pas totalement disparu. En témoignent les entraides coutumières encore vivaces dans certains villages. L'individualisme foncier a fait des paysans descendants des lignages fondateurs des villages et membres des ethnies autochtones, des propriétaires de terre.

\section{Les pratiques foncières}

En conséquence de cette évolution de la situation foncière, il existe aujourd'hui plusieurs modes d'accès à la terre dont les uns sont relativement anciens, et les autres plus récents.

\section{Les modes anciens d'accès à la terre}

Ils sont au nombre de trois : l'héritage, le don, l'emprunt.

Le premier et le plus important est l'héritage. Quand un chef de famille meurt, ses enfants héritiers se partagent ou se font partager son domaine foncier. Les enquêtes que nous avons menées entre 1993 et 1995 (Klassou, 1996, p. 286-292 ; Abotchi, 1997, p. 112-118) ont révélé que ce mode d'accès à la terre n'est prépondérant que dans les villages peuplés majoritairement d'autochtones. A Ahassomé, en milieu adja, et à Hodikou, en milieu éwé, il concerne respectivement $58 \%$ et $60 \%$ des terres (fig. 2). Il est même plus important à Adogbénou en milieu ana ou il concerne $69 \%$ des parcelles foncières. La faible part des terres héritées à Notsé (37\%) tient au fait que Notsé étant une ville, beaucoup de ses habitants sont des «étrangers » mais qui complètent leurs activités principales de salariés, employés ou artisans, par des activités agricoles. 
Figure 2. Régime des terres dans les villages enquêtés.

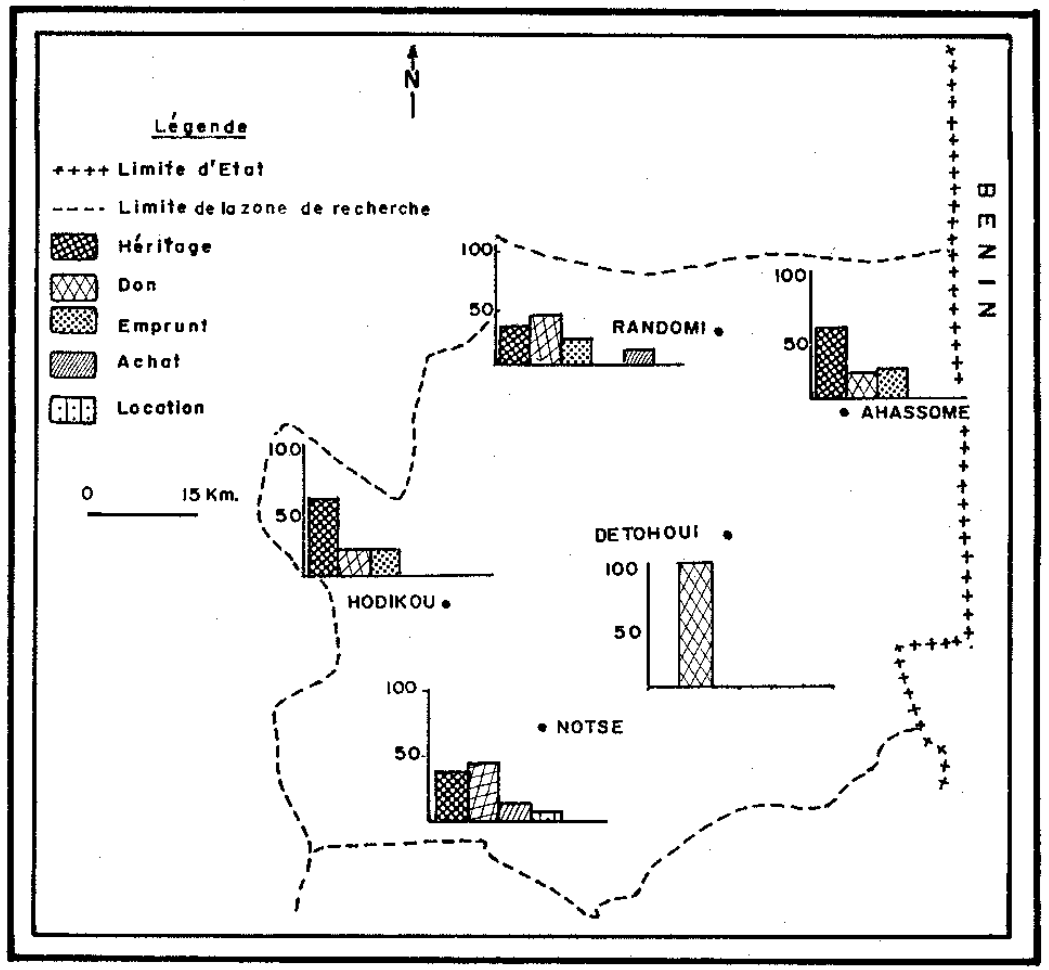

Il convient de faire remarquer que, dans notre région, seuls les hommes héritent des terres de leur lignage ou de leur père, soit sous la forme d'un usufruit permanent dans le cas de la propriété collective, soit sous celle de la propriété individuelle lorsqu'il y a partage du domaine foncier familial. Il s'agit là d'une règle coutumière que ni l'évolution économique récente, ni l'étonnante liberté économique actuelle de la femme adja-éwé, n'ont réussi à effacer. La femme adja-éwé dispose en effet de son propre champ qu'elle gère en toute liberté et dispose aussi pleinement du produit de ses activités commerciales ou artisanales. Mais lors du partage de la terre, les filles sont exclues de l'héritage alors que les ayants droit déjà morts ont droit à leur part s'ils ont eu une progéniture mâle. Cette pratique n'est pas liée, comme c'est le cas chez les Ouéménou du Bénin, au départ des filles de leur concession natale au moment du mariage (Pelissier, 1962, p. 249). Elle résulte du fait que, dans la société adja-éwé, les enfants appartiennent au lignage de leur père. Si la femme héritait des terres de son patrilignage, ces terres reviendraient à sa mort à ses enfants. Or si son mari appartenait à un autre lignage, il y aurait transfert des terres à ce dernier, ce qui serait intolérable au regard de la coutume.

Traditionnellement donc, la succession est de type patrilinéaire, avec seulement un droit de culture pour la femme. Mais il existe cependant des femmes qui deviennent propriétaires de terres, soit en les achetant, soit en contournant la pratique coutumière en s'en faisant donner par leur père du vivant de celui-ci. Certaines les lèguent alors à leur descendance féminine, leurs fils n'ayant qu'un simple droit d'usufruit. Ces terres qui se transmettent par la branche féminine (Abotchi, 1997, p. 114) sont de dimensions variables mais le plus souvent de petite taille et ne présenteraient aucun intérêt à être 
partagées. Elles ont résisté plus que toutes les autres, aux changements. Jusqu'aujourd'hui encore, elles ne donnent lieu ni à partage, ni à aliénation.

Comme on le voit, en ce qui concerne la transmission des droits de propriété, deux types de terre sont ici présents: les terres de la branche masculine, le type le plus répandu, et les terres de la branche féminine, plus rares.

En deuxième lieu, il y a le don. Le chef de famille peut, pour des raisons diverses et dans des circonstances qu'il serait fastidieux d'énumérer ici, donner une ou des parcelles de terre à des personnes qui ne sont pas membres de sa famille mais appartiennent à d'autres lignages ou qui sont étrangères à la communauté villageoise, parfois même membres d'autres groupes ethniques. Selon nos enquêtes en 1994 dans cette plaine du Mono, les terres acquises par don dominent dans les secteurs peuplés à majorité d'immigrants de fraîche date (Abotchi, 1997). Cependant, à Détohoui (préfecture de Haho), où toutes les terres semblent relever du don (fig. 2), il faut peut-être réduire quelque peu leur part car les termes «don» et "prêt » étant homonymes dans le langage local, nos enquêteurs ont pu les confondre et tout ranger dans la catégorie «don ». Eu égard à l'attachement à la terre dans notre région d'investigation, à la proscription culturelle de l'aliénation de la terre, mais aussi en raison de la pression démographique résultant de l'immigration des populations éhoué du Bénin et kabyélosso du Nord-Togo, et d'un croît naturel élevé, ce qui a fait naître une crise d'espace agricole dans certains secteurs (Abotchi, Aflou, Akibode, 1999), on peut admettre que le don de terre n'a jamais été une pratique courante. Au cours de ces dernières décennies, le problème de manque de terre se posant avec acuité, plus personne ne donne de la terre à des étrangers, la tendance étant même à la reprise des terres déjà cédées.

Le troisième mode d'accès à la terre est l'emprunt, qui s'impose aujourd'hui comme la pratique la plus courante pour les étrangers qui veulent s'installer dans le village ou pour les autochtones qui veulent ouvrir de nouveaux champs. Pour l'obtenir, il faut en faire la demande à un propriétaire de terre en lui offrant une ou deux bouteilles de sodabi (alcool local, provenant le plus souvent de la distillation du vin de palme) et parfois en plus, du tabac. D'autre part l'emprunteur devra au prêteur une partie de sa production annelle, un sac de maïs, et une certaine somme d'argent. C'est à ce système qu'ont recours aujourd'hui la quasi-totalité des immigrants qui s'implantent dans la région.

26 A l'inverse de l'héritage et du don qui permettent d'acquérir un droit définitif de propriété, l'emprunt n'autorise, quant à lui, qu'un droit de culture temporaire, la terre devant retourner au propriétaire lorsque celui-ci voudra la récupérer pour son propre usage ou pour un membre de sa famille. Bien que l'emprunt soit un mode d'accès à la terre souple et qu'il permette ainsi à tout le monde d'accéder à la terre, il présente néanmoins pour le développement rural des inconvénients, dont deux méritent d'être signalés. D'abord, le fait que la terre puisse être retirée à tout moment crée pour l'exploitant une insécurité foncière qui ne l'incite ni à des pratiques de protection ou de conservation du sol ni à des investissements susceptibles de l'améliorer. Le paysan ne voudra pas par exemple fertiliser une terre qu'il n'est pas sûr de pouvoir cultiver l'année suivante. "Comment un agriculteur pourrait-il investir dans son champ du travail et du capital s'il n'en est pas propriétaire? » se demandait à ce propos Gourou (1971). Comme le souligne Tcherkezoff (1992 p.124), "tout investisseur, individu ou société, recherchera un contrat garantissant à long terme son droit d'usage ou accordant une autorité conjointe sur cette terre». Comme dans notre région cette 
garantie n'existe pas, l'exploitant à statut précaire risque d'exploiter abusivement le sol et de le dégrader. Le système du prêt coutumier de la terre est un obstacle à certains types d'activité économique (agriculture intensive, plantations arbustives, par exemple ), et par conséquent un obstacle au développement rural.

Le second inconvénient de ce mode d'accès au sol est le fait qu'il limite, pour l'exploitant, le choix des cultures. En règle générale, seules les cultures annuelles sont autorisées. Les arbres fruitiers comme le palmier à huile, le manguier et l'oranger, et les autres arbres utiles tels que le teck et l'eucalyptus, sont proscrits. "Amédjro mé doa ati alo édétio o " (un étranger ne plante pas d'arbre ou de palmier) dit-on dans la région. Ici, en l'absence d'écriture en milieu rural, l'arbre planté (palmier à huile, le teck, etc.) et aussi toutes les autres cultures pluriannuelles sur un terrain marquent le droit de propriété (Klassou, 1996, p. 290 ; Abotchi, 1997, p. 175). Les prêteurs entendent ainsi éviter de se faire déposséder de leur terre à terme, mais ce système pénalise fortement les exploitants qui ne tirent pas plein profit des parcelles qui leur sont octroyées. Parfois cependant, des arrangements sont trouvés entre propriétaire et exploitant, qui leur permettent de partager la production des arbres qui seraient plantés par l'exploitant (à raison de 1 contre 1 ou 2 contre 1 selon les cas - dans d'autres régions du Togo, dans le Litimé au sud-ouest par exemple, des contrats «madibi » partagent la propriété de la terre et des arbres entre le prêteur et l'exploitant). Souvent le propriétaire est le plus gros bénéficiaire de l'opération. En pays adja surtout, dans le Moyen-Mono et dans l'Est de la préfecture de Haho, lorsqu'un paysan prête une terre à une autre personne qui l'exploite en pratiquant des cultures annuelles, il y plante en association des palmiers qui non seulement rappellent son droit de propriété mais de plus lui assurent le retour de la terre dans un temps proche, cinq à huit ans, lorsque les palmiers devenus grands ne permettront plus, en raison de leur ombrage, de cultiver les plantes saisonnières (Lange, 1986, p.11). Or le palmier rapporte gros. Il joue en outre un rôle socio-culturel important : il intervient par ses sous-produits dans diverses cérémonies traditionnelles et constitue une épargne qui peut être mobilisée en cas de besoin et une caisse de retraite pour les personnes âgées. C'est donc une culture qui n'est pas permise au non-propriétaire.

Héritage, don et emprunt constituaient les voies par lesquelles on accédait à la terre. Mais récemment, avec l'évolution de l'économie, de nouveaux modes sont apparus.

\section{Les modes d'accès à la terre d'origine récente}

Il s'agit de la location, de la mise en gage et de l'achat. Ces trois modes sont antinomiques des règles coutumières qui interdisent formellement que la terre fasse l'objet de transaction monétaire, mais ils se généralisent largement aujourd'hui en conférant de plus en plus de valeur à la terre au fil des années. Cette valeur est variable d'un cas à l'autre et d'un secteur à un autre. Dans l'est de la préfecture de Haho, entre Tététou et Kpové, le prix du loyer annuel est d'un sac de maïs et $5000 \mathrm{~F}$ CFA, soit 7,62 euros (100 F CFA = 0,152 euro) par personne quelle que soit la superficie louée. A Djadéhoé, il s'élève à 10 000F CFA par an (Abotchi, 1997) et à Alati, le paysan exploitant moins de 5 ha doit au propriétaire, et par an, $10000 \mathrm{~F} \mathrm{CFA} \mathrm{et} \mathrm{un} \mathrm{sac} \mathrm{de} \mathrm{produit} \mathrm{récolté,}$ ainsi qu'un coq, des ignames et une bouteille d'alcool importé (gin, rhum, whisky,...) pour les fêtes de fin d'année. A Kpékplémé dans la préfecture du Moyen-Mono, le loyer est compris entre 20000 et 30000 F CFA par hectare. Quant au prix d'achat de la terre, il varie suivant plusieurs facteurs: situation topographique du terrain, qualité 
agronomique du sol, distance par rapport au village ou aux axes de communication, etc. En 1995, il était compris entre 200000 et 300000 F CFA par hectare dans la région de Kpékplémé.

Il faut cependant signaler que, comme l'a aussi observé Tallec (1986), les paysans parlent difficilement de la location de la terre, encore moins de la vente. La monétarisation de la terre est un sujet tabou car c'est un interdit culturel très strict. Les paysans craignent que la non-observance des pactes telluriques conclus par leurs ancêtres avec les divinités chthoniennes n'entraîne une malédiction qui attirerait sur eux une punition immanente parfois irréversible. Ils refusent donc d'admettre ou de dire qu'ils effectuent des transactions sur les terres, en prétendant même que les frais de location ne sont qu'un simple témoignage de reconnaissance de la part des locataires.

31 Quant au gage enfin, il n'est pas très répandu mais il existe et peut concerner, soit une terre que le créancier peut exploiter, soit une palmeraie que porte la terre, soit enfin les deux à la fois. Mais comme le créancier qui ne peut se faire rembourser la somme prêtée au moment où il a à son tour besoin d'argent, peut, comme c'est aussi le cas dans la société ouéménou au Bénin (Pelissier, 1962, p. 253), « céder son gage (ou une partie de celui-ci) à un tiers qui endosse ses droits en lui avançant son prêt, la situation de certaines parcelles mises en gage semble extrêmement complexe ou de moins apparaît comme très compliquée ". Comme l'emprunteur ne parvient pas toujours à rembourser, le gage se présente alors comme un prélude à la vente de la terre, à un transfert définitif au prêteur.

\section{Les causes de l'évolution du système foncier}

32 A l'origine de cette évolution du système foncier qui conduit au viol des interdits coutumiers, nous retiendrons particulièrement deux causes. La première est la faiblesse des ressources financières des paysans par rapport aux multiples charges auxquelles ils doivent faire face. Ils sont aujourd'hui en tout cas assaillis par d'importants besoins d'argent liés à la survivance des obligations coutumières, en particulier les mariages et les funérailles, qui sont célébrés en grandes pompes avec d'interminables manifestations auxquelles sont conviées à manger et à boire plusieurs centaines de personnes et qui s'étendent parfois sur plusieurs semaines, et que certains se plaisent même à renouveler périodiquement pour leur honneur ou pour obtenir la bénédiction des ancêtres. Le montant de la dot, c'est-à-dire de la compensation de mariage, a aussi flambé. Jadis symbolique, il atteint aujourd'hui plusieurs centaines de milliers de francs CFA. Dans sa composition même, les alcools importés remplacent désormais le sodabi et surtout le vin de palme, qui n'apparaît plus que de façon symbolique. Les bijoux de grand luxe (en or notamment) et les tissus d'apparat (le wax hollandais en particulier) sont exigés en plusieurs exemplaires, 6 à 12 selon les cas (d'une demi-pièce chacun, or une pièce de wax hollandais se vend aujourd'hui entre 60000 et 80000 F CFA), Par ailleurs, des dépenses importantes concernent aussi les soins de santé, la scolarisation des enfants,... et l'achat des splendeurs matérielles de la civilisation européenne qui fascinent énormément (biens d'équipement et de prestige). Beaucoup de parents ont deux ou trois enfants à l'école primaire ou au collège ; beaucoup achètent des postes radio, des radiocassettes, des vélos et des motos, des meubles, du ciment et de la tôle 
ondulée pour la construction de l'habitation, etc. Pour satisfaire tous ces besoins d'argent, les paysans louent, mettent en gage ou vendent leurs terres à très bas prix.

La deuxième cause est liée à l'action des églises chrétiennes. C'est dès le début du $\mathrm{XX}^{\mathrm{e}}$ siècle que les missionnaires catholiques ont commencé à effectuer des visites d'évangélisation dans la région à partir de Nuatja (Notsé) et de la subdivision de Parahoué (dans l'ex-Dahomey). Les rapports des administrateurs coloniaux leur avaient montré que, bien que la région fût difficile d'accès avec une jungle épaisse sans véritable voie de communication, où pullulaient moustiques, glossines et simulies, il $\mathrm{y}$ vivait cependant de nombreux indigènes, c'est-à-dire des âmes à conquérir au Christ. Mais malgré un grand travail de conversion dans les années 1940 et 1950 encore, l'emprise du christianisme était restée faible.

Depuis deux ou trois décennies, on assiste par contre à une explosion de dynamisme des religions importées. Le catholicisme d'abord, puis l'Église Évangélique, et récemment le pentecôtisme, l'Église des Assemblées de Dieu et l'Église Baptiste. En 1981 déjà, on recensait quelque 28479 adeptes des religions étrangères contre 57361 animistes. Depuis dix ans environ, ces religions étrangères connaissent une affluence impressionnante à tel point que les chrétiens formeraient aujourd'hui la communauté religieuse numériquement la plus importante.

Mais ces religions chrétiennes, en particulier les plus nouvellement implantées (Église des Assemblées de Dieu, Église Baptiste, Église de Pentecôte, Église du Christianisme Céleste, etc.) sont très fondamentalistes et rejettent catégoriquement toute compromission avec les croyances ancestrales. La conversion à ces religions étrangères conduit donc au rejet des croyances et pratiques coutumières qui constituent le fondement même des règles qui régissent l'usage et le transfert de la propriété de la terre en droit traditionnel. En particulier sont ignorées par les chrétiens les malédictions des dieux et des ancêtres qui constituaient les seules forces coercitives contre le non-respect des pratiques coutumières, avec bien sûr les pesanteurs sociales qui faisaient de la vente de la terre une déviance morale (Aflou, 1998, p. 43-46). La poussée actuelle du christianisme dans la région favorise donc la monétarisation de la terre et l'évolution du système foncier.

\section{Les conséquences de l'évolution du régime foncier}

L'évolution du système foncier en milieu adja-éwé s'accompagne d'importantes conséquences dont trois au moins méritent d'être signalées ici. En premier lieu, le partage des domaines lignagers ayant conduit à des propriétés individuelles de petite taille, la diffusion de l'attelage et du tracteur en milieu paysan est difficile. En effet, pour accéder à ces outils de travail modernes, le paysan doit disposer au minimum de 5 ha pour la culture attelée, de 15 à 20 ha pour la culture motorisée. Dans notre région caractérisée par un émiettement foncier, par exemple 8 à 100 ares par propriété dans le canton de Kpékplé-mé (Abotchi, Aflou, Akibode, 1999, p.33), le manque de terre constitue assurément une contrainte limitative à l'adoption de l'attelage et du tracteur. D'après une étude menée en 1994 dans l'Est de la Région des Plateaux (Oladokoun, 1995), $20 \%$ des paysans en culture manuelle affirment en effet identifier le manque de terre comme obstacle à l'acquisition de la traction bovine. 

friche qui ne peuvent être exploitées efficacement par leurs propriétaires, alors qu'ailleurs des ménages végètent sur des terres exiguës et surexploitées (Ereou, 1998, p. 26). émiettée et contrastée. C'est cette double constatation sur le plan national ainsi que les contraintes qui en découlent pour la production agricole, qui ont amené les autorités à faire promulguer en 1974 une réforme agro-foncière "qui préconise de rendre obligatoire l'exploitation communautaire des terres au sein de grandes coopératives de production » et qui dispose que «les terres incultes jusqu'à ce jour (c'est-à-dire les terres n'ayant fait l'objet d'aucune mise en valeur depuis plus de 10 années consécutives) appartiennent à la nation dont elles constituent le domaine foncier national, terres que l'État pourra redistribuer sous toutes les formes qu'il lui appartiendra de déterminer " (Bertelot, de Ravignan, 1980, p. 60). Mais cette réforme est restée sans effet véritable, à cause des difficultés de son application.

deuxième lieu, l'apparition du système de vente engendre des transferts de propriété, surtout des paysans autochtones à des gens d'origine étrangère à la région. Sont notamment acquéreurs des terres par achat auprès des paysans adja et éwé du Moyen-Mono et du Haho, les commerçants installés dans les grandes villes du pays, les populations éhoué du Bénin. Celles-ci viennent du plateau béninois d'Aplahoué depuis le XIX siècle (certains groupes étaient sans doute arrivés déjà précédemment pour fuir les armées esclavagistes d'Agbomé) mais le mouvement était devenu massif depuis la fin des années 1920 pour de multiples raisons (fuir les obligations de service militaire au Dahomey, différences de taxes entre les deux pays liées à leur statut international différent, différences qui se sont maintenues parfois après l'accession à l'indépendance, recherche de terres nouvelles et meilleures pour la culture que celles de leur plateau d'origine, etc. - sur ce sujet, voir Abotchi, 1995, 1998). Ont été aussi acquéreurs de terres les hommes politiques habitant dans les grandes villes auxquels on pourra ajouter quelques notables locaux "puissants» (Abotchi, 1997, p. 135-136; Klassou, 1996, p. 291). C'est ainsi que des propriétés de plusieurs dizaines d'hectares appartiennent aujourd'hui à des personnes issues de la classe politique du pays et de la bourgeoisie urbaine, qui résident généralement à Lomé ou à Atakpamé, et qui exploitent de grandes fermes utilisant du matériel agricole moderne (tractions motorisée et bovine) et emploient quelques ouvriers et un contremaître. Ces nouveaux propriétaires n'hésitent pas à faire appel à des géomètres pour l'abornement de leurs terres et pour les enregistrements légaux de leurs acquisitions.

C'est ainsi que de plus en plus de paysans cèdent facilement leurs terres contre quelques milliers de francs CFA et se retrouvent obligés d'en emprunter ou d'en louer auprès des propriétaires qui en ont encore, ou de se faire engager par leurs voisins comme ouvriers agricoles payés à la tâche ou à la journée. Mais les salaires agricoles sont faibles, en moyenne $500 \mathrm{~F}$ CFA la journée. Avec de tels salaires, et en l'absence de "grenier pour soi », c'est-à-dire d'économies, il leur est difficile de subvenir financièrement à leurs besoins toute l'année (notamment pendant la saison morte, entre décembre et février) et d'assurer de façon plus ou moins satisfaisante, la soudure alimentaire entre avril et juillet. Aussi se retrouvent-ils aux prises avec de sérieuses difficultés économiques et financières, en même temps que déconsidérés en raison des pesanteurs sociales par suite de la vente de leurs terres. La misère et surtout les regards 
inquisiteurs de la communauté villageoise leur devenant insupportables, ces expropriétaires terriens migrent vers les villes où ils se font herboristes guérisseurs ou devins, la majorité de la population ne pouvant plus par suite de la crise actuelle, se faire soigner dans les hôpitaux. Ou bien ils se font employer dans les métiers à bas statut social et peu rémunérateurs : gardien, aide maçon, etc. (Klassou, 1996, p. 291). Leur vie y est donc généralement misérable.

Comme on le voit, non seulement les ventes de terre font naître des problèmes sociaux dans les campagnes, mais elles contribuent aussi à aggraver les problèmes en milieu urbain.

Par ailleurs, avec les systèmes de location et surtout de vente, la terre commence à prendre une valeur et une finalité nouvelles. Désormais elle n'est plus seulement un moyen de survie et de reproduction du groupe social et de ses éléments constitutifs, mais aussi un bien accumulable et une source de richesse. Aussi la terre est-elle devenue un véritable enjeu, les propriétaires prenant conscience de la raréfaction des terres due à la pression démographique et des transferts massifs qu'ils ont effectués à leurs propres dépens. Il en résulte de nombreux conflits.

Ces conflits naissent en général des problèmes de partage et de délimitation des domaines familiaux, et de contestation des limites des parcelles; ils naissent aussi des tentatives de reconquête foncière de la part de certains propriétaires et surtout de leurs descendants qui veulent récupérer des terres données jadis verbalement en usufruit, prêt ou location à des personnes qui se les sont appropriées. Le caractère flou des limites des parcelles, l'absence de contrat de vente ou de cession de terre, le manque de titre de propriété ou d'enregistrement cadastral, et des divergences dans l'interprétation des transferts de terres par les propriétaires coutumiers aux immigrants, sont, entre autres, les facteurs expliquant la multiplicité de ces conflits fonciers (Abotchi, Aflou, Akibode, 1999, p. 35 ; Klassou, 1996).

Ces conflits peuvent entraîner de sérieux problèmes de cohabitation dans certains villages. C'est le cas à Kpoguédé, à côté de Tététou, où les Ehoué regroupés dans le quartier Ngbonfoui et les Adja rassemblés dans le quartier Totouhon vivent en permanence dans un climat de tension qui se ravive et s'apaise sporadiquement. C'est également le cas à Asrama entre les Éhoué de Kanmé et les Adja d'Asrama-marché, et à Kpové-Djémégni, près de Notsé, entre les Éwé et les Éhoué (Abotchi, 1997, p. 303). Ces conflits placent aujourd'hui la question foncière au coeur des problèmes de développement rural.

\section{Conclusion}

45 Jadis propriété des ancêtres, moyen de survie et de reproduction du groupe social, indivise et inaliénable, la terre était distribuée gratuitement selon les normes coutumières et exploitées dans le cadre de propriétés collectives régentées par les chefs de lignage. Mais les transformations sociales et économiques introduites depuis l'époque coloniale ont entraîné des changements importants. La terre fait l'objet de toutes sortes de transactions et prend de plus en plus de valeur monétaire au fil des années. Les conséquences de cette situation sont variées et parfois fâcheuses sur le plan du développement. 

vente de la part des anciens propriétaires (autochtones), ne sont pas seulement en rapport avec la valeur monétaire que ces terres prennent et avec leur rareté ; elles sont aussi et surtout liées à l'attachement à la terre, attachement dû à sa valeur culturelle : céder une terre apparaitt comme perdre son identité, perdre ses repères historiques. Il apparaît ainsi que malgré les évolutions, la terre garde dans notre région d'investigation sa valeur culturelle. On comprend alors que la cession à titre définitif de la terre ne soit pas encore totalement acceptée par les Ewé et les Adja, propriétaires coutumiers du sol. On voit par là que la région se trouve dans une situation de transition inconfortable.

Quoi qu'il en soit, la terre a aujourd'hui une valeur et une finalité telles qu'elle est l'enjeu de rivalités entre lignage, famille et individu qui enveniment les relations sociales dans les villages. Les conflits fonciers sont si graves qu'il conviendrait de les arbitrer très rapidement et de façon durable si l'on ne veut pas que les divergences politiques nées du multipartisme les exacerbent en créant une situation explosive dans les campagnes.

\section{BIBLIOGRAPHIE}

ABOTCHI T. (1995), «L’immigration des Éhoué du Bénin dans la plaine du Togo. Le cas du Habo oriental », Les Cahiers d'Outre-Mer, 192, pp. 453-475.

ABOTCHI T. (1997), Dynamisme économique et évolution du milieu rural dans l'est de la Région des Plateaux au Togo, Thèse de Doctorat de Géographie et Gestion des espaces, Université de Bordeaux 3, $377 \mathrm{p}$.

ABOTCHI T. (1998), « Réussite économique et mutations sociales d'une communauté d'immigrants : Les Éhoué du Haho oriental et du Moyen-Mono au Togo », Annales de l'Université du Bénin, série Lettres, Tome XVIII, Lomé, pp. 72-107.

ABOTCHI T., AFLOU D., AKIBODE K.A. (1999), «Crise de l'espace agricole et développement rural au Togo : cas du canton de Kpékplémé », Bulletin de la Société Neuchâteloise de Géographie, $n^{\circ} 42-43$, 1998-1999, pp. 31-56.

AFLOU D. (1998), Le problème de la raréfaction des terres destinées aux cultures vivrières dans le canton de Kpékplémé (Préfecture du Moyen-Mono), Mémoire de Maîtrise de Géographie rurale, Université du Bénin, Lomé, 148 p.

ALLA DELLA A. (1997), « Les enjeux fonciers de l'économie de plantation dans le centre-ouest de la Côte d'Ivoire : l'exemple du pays bété de Daloa ", Africaines, $n^{\circ}$ 4, pp. 43-56.

BERTHELOT J. \& de RAVIGNAN F. (1980), Les Sillons de la Faim, Collection alternatives paysannes, L'Harmattan, Paris, 223 p.

CORNEVIN R. (s.d.), « Les Éhoué d'Akplahoué dans l'Est-Mono togolais », in Les migrations internationales de la fin du XVIII siècle à nos jours, CNRS, Paris, pp. 227-239. 
EREOU K. (1998), La commercialisation des produits agricoles dans la région de Haho et ses impacts sur le niveau de vie des paysans, Mémoire de Maîtrise de Géographie, Université du Bénin (UB), Lomé, 124 p. + annexes.

GAYIBOR N.L. (1990), « Kpoyizu ou la fin d'une époque », Annales de l'UB, Série Lettres, Tome XI, Lomé, pp. 25-41.

GLOWCZEWSKI-BARKER B. (1992), « La terre, ma chair (Australie) », Études rurales, 127-128, juilletdécembre, pp. 89-105.

GOUROU P. (1971), Leçons de géographie tropicale, Paris, 323 p.

GU-KONU E.Y. (1986), « Une pratique foncière dans le sud-ouest du Togo : Le dibi-madibi », in Espaces disputés en Afrique noire, Pratiques foncières locales, Ed. Karthala, Paris, pp. 243-252.

KLASSOU K.S. (1996), Évolution climato-hydrologique récente et conséquences sur l'environnement. L'exemple du bassin versant du fleuve Mono (Togo-Bénin), Thèse de doctorat de Géographie et Gestion des espaces, Université de Bordeaux 3, 467 p.

LANGE M.F. (1986), « Dynamisme économique et reviviscence sociale et culturelle chez les AdjaÉhoué du Moyen-Mono (Togo). Quel projet de société ? ", Communication au Colloque Terrains et perspectives, ORSTOM, Lomé, 23 p.

LOMBARD J. (1961), « Les bases traditionnelles de l'économie rurale Bariba et ses fondements nouveaux. Conclusions à une enquête effectuée dans la région de Bembéréké (Haut Dahomey) », Bulletin de l'IFAN, Tome XXII, Série B, 1-3, pp. 199-242.

OLADOKOUN W. (1995), La dimension sociale et économique de la culture cotonnière au Togo (Cas de la Région des Plateaux), Mémoire de Maîtrise de Géographie, UB, Lomé, 108 p. + annexes.

PÉLISSIER P. (1962), « Les pays du Bas Ouémé. Une région témoin du Dahomey méridional », Les Cahiers d'Outre-Mer, 59, pp. 204-254.

SURGY (de) A. (1996), « La multiplicité des Églises au sud de l'Afrique occidentale », Afrique Contemporaine, 177, pp. 30-44.

TALLEC M. (1986), Étude de la diversité des systèmes de production dans la région de Notsé au Togo, Mémoire ENSSAA/ESAT, CIRAD-DSA/IRAT, Dijon, Montpellier, Kolocopé (Anié), 135 p.

TCHERKEZOFF S. (1992), « Les enfants de la terre aux îles Samoa : Tradition locale et “développement" importé ", Études rurales, 127-128, pp. 15-40.

\section{RÉSUMÉS}

Les préfectures de Haho et du Moyen-Mono au Togo sont caractérisées par des modes de gestion foncière qui reflètent les croyances coutumières des populations et les rapports qu'elles entretiennent avec leur environnement. Mais depuis l'avènement du système colonial et l'expansion de l'économie monétaire apparaissent de nouvelles pratiques foncières qui se développent de plus en plus, et qui, tout comme les modes traditionnels de cession de la terre, ne manquent pas de poser de multiples problèmes à la croissance agricole et au développement rural.

Haho and Moyen-Mono prefectures in Togo are characterised by traditional land distribution systems that reflect the customary beliefs of the populations and their relations with the environment, as is the case elsewhere in Tropical Africa. But the advent of the colonial system and the development of a monetary economy have generated increasing new land practices. Both 
the traditional land distribution system and these new practices pose many problems to agricultural growth and rural development.

\section{INDEX}

Mots-clés : Togo, préfecture de Haho, préfecture du Moyen-Mono, croyances coutumières, pratiques foncières, développement rural

Keywords : Togo, prefecture of Haho, prefecture of Moyen-Mono, customary beliefs, land distribution practices, rural development

\section{AUTEUR}

\section{SELOM K. KLASSOU}

Département de Géographie, Faculté des Lettres et Sciences Humaines, Université du Bénin, Lomé (Togo) 